\title{
Spontaneous C1-2 CSF Leak Treated with High Cervical Epidural Blood Patch
}

\author{
Philip WH Peng, Richard Farb
}

Can. J. Neurol. Sci. 2008; 35: 102-105

Spontaneous intracranial hypotension $(\mathrm{SIH})$ is a condition caused by spontaneous spinal cerebrospinal fluid (CSF) leaks. ${ }^{1}$ Clinically, it is characterized by orthostatic headache and may respond well to epidural blood patch. ${ }^{2}$ Other neurological presentations of SIH include diplopia, memory loss, hearing deficits, Parkinsonism, ataxia, obtundation and even coma. ${ }^{1}$

Magnetic resonance imaging (MRI) is crucial in the diagnosis of this condition. Typical radiological features include diffuse pachymeningeal enhancement, descent of the cerebellar tonsil, a decrease in the size of prepontine and perichiasmatic cisterns, and subdural fluid collections. ${ }^{3}$ However, radiological imaging such as radionuclide cisternography or spinal MRI are not reliable in detecting the site of leakage. ${ }^{1,4}$ Myelography with iodinated contrast followed by thin-cut computed tomography (CT) or MRI of the entire spine has been shown as the technique of choice in defining the location of the CSF leak. ${ }^{1,5,6}$
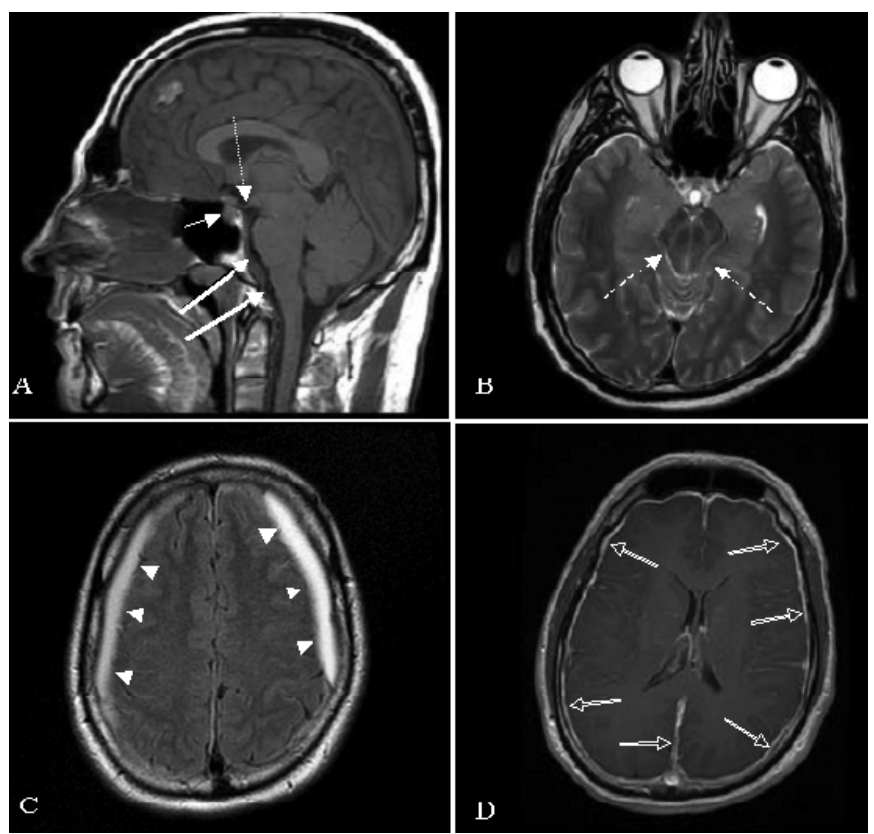

The management of CSF leakage at the low cervical level with an epidural blood patch has been reported..$^{-9}$ However, leakage at C1-2 is usually managed with surgical repair. ${ }^{10-11}$ One case report described the use of an epidural blood patch at the $\mathrm{C} 2$ level performed under CT guidance. ${ }^{12}$ We report a case in which a patient with CSF leakage at C 1-2 was successfully treated with a high cervical epidural blood patch under fluoroscopic guidance. This case report also demonstrates that intrathecal administration of gadolinium (gadopentetate dimeglumine) can be a useful alternative to iodinated contrast in patients with true allergy to iodine.

\section{CASE history}

A 39-year-old man with the diagnosis of spontaneous intracranial hypotension was referred for an epidural blood patch. He presented with a two-month history of orthostatic

Figure 1. Typical MR findings in intracranial hypotension. A) midline sagittal T1WI showing the "sagging brain" appearance with distortion of the anterior margin of the pons and medulla (long arrows), decreased vertical dimension of the suprasellar cistern and sagging tuber cinerium (dotted arrow) as well as the engorged pituitary gland (short arrow). B) axial T2WI showing effacement of the perimesencephalic cistern (dashed arrows) as well as the elongated AP dimension of the midbrain. C) axial FLAIR image showing small bilateral subdural fluid collections (arrowheads) with increased signal intensity indicating previous subdural hemorrhage. D) gadolinium enhanced axial T1WI demonstrating diffuse pachymeningeal (dural) enhancement (open arrows).

\footnotetext{
From the Department of Anesthesia (PWHP) and Radiology (RF), Toronto Western Hospital, University Health Network, University of Toronto, Toronto, Ontario, Canada. Received June 27, 2007. Final Revisions Submitted October 30, 2007.

Reprint requests to: Philip Peng, Department of Anesthesia and Pain Medicine, McL 2-405, Toronto Western Hospital, 399 Bathurst Street, Toronto, Ontario, M5T 2S8, Canada.
} 

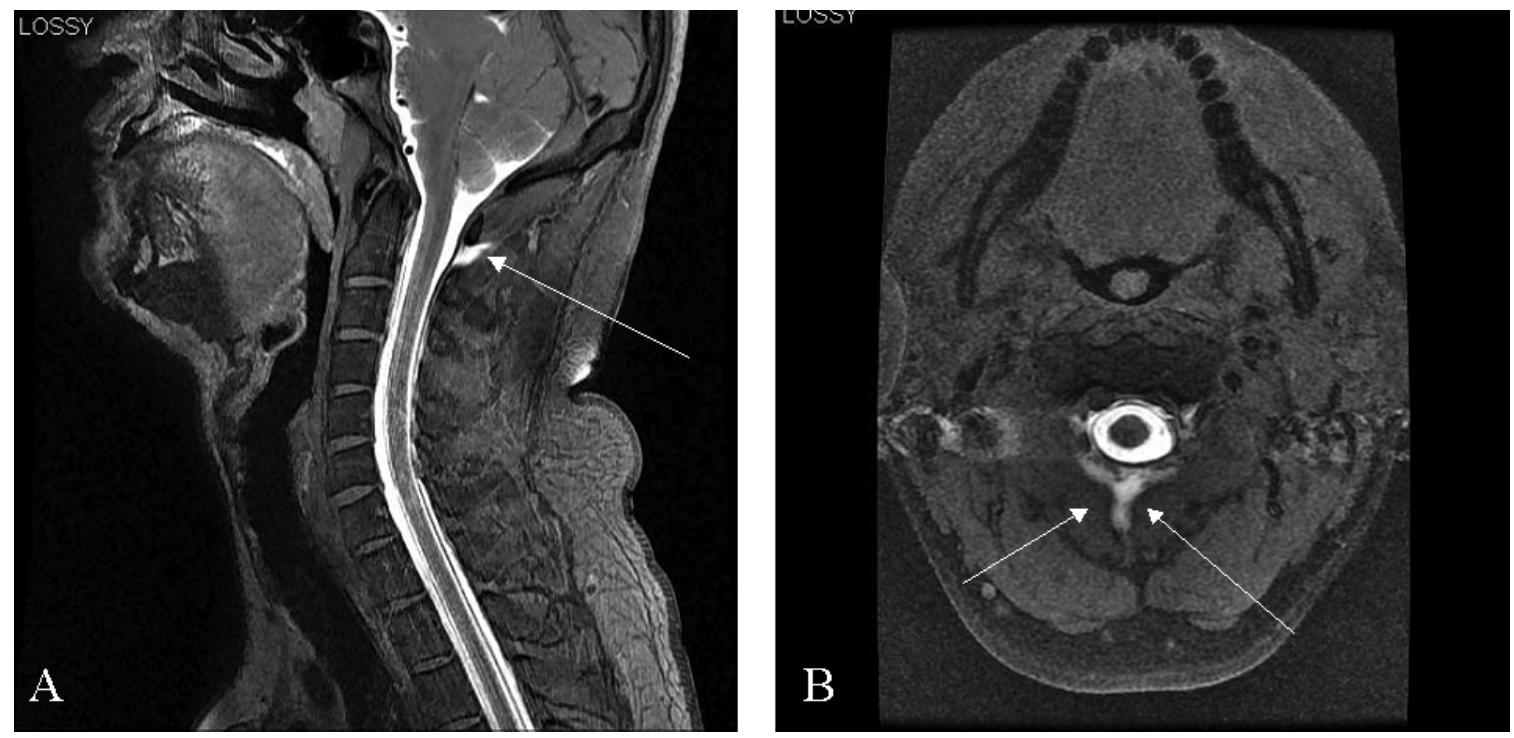

Figure 2. A and B. Sagittal and axial T1 weighted fat saturated images obtained after intrathecal injection of gadolinium. Note the small amount of leakage of gadolinium enhanced CSF into the posterior paraspinal soft tissues (arrows) at the C2 level.

headache without any associated neurological symptoms. An MRI revealed features suggestive of SIH (Figure 1A-D). Previously, he underwent a spine MRI which failed to localize the site of leakage. As the common site for a CSF leak is usually at the thoracolumbar junction, an epidural blood patch was performed at the L1-2 level with $20 \mathrm{ml}$ of autologous blood. The patient failed to respond to this injection. In our institution, our experience suggested that radionuclide cisternography had not been reliable in detecting the level of the leak. Because of the patient's history of allergy to iodinated contrast media (documented anaphylactic reaction), we decided not to perform CT myelography at that time. A second attempt of epidural blood patch was performed at the T5-6 level. Again, he failed to respond and started to experience ataxia. After a discussion with the neuroradiologist, the patient decided to receive a spine MRI with an intrathecal injection of gadolinium (Magnevist; Schering, Berlin, Germany), an off-label use of this contrast. ${ }^{13,14}$ Following the insertion of a $22 \mathrm{G}$ spinal needle at L2-3 level, 3 $\mathrm{ml}$ of CSF was aspirated and mixed with $0.5 \mathrm{ml}$ of gadolinium. The mixture was then slowly reinjected intrathecally over one minute. The MRI successfully revealed the leakage site at the C1-2 level (Figure 2A,B).

Because of the proximity of C1-2 level to the 'sagging' brain stem, an epidural blood patch at C3-4 was proposed to the patient. The procedure was performed with the patient in the prone position with fluoroscopic guidance. A 17 gauge Tuohy epidural needle was inserted at the C3-4 level at midline and the epidural space was localized with hanging drop technique, in which the epidural needle was filled with normal saline and the meniscus of fluid level could be seen at the hub of the needle. When the tip of the epidural needle entered the epidural space, the meniscus disappeared due to the negative pressure of the epidural space. A total of $15 \mathrm{ml}$ of autologous blood was obtained from the dorsal vein of the right hand under sterile technique and injected into the epidural space. The patient tolerated the procedure well without any localized or referred discomfort.

The injection resulted in gradual improvement of the patient's headache, and by day seven his headache was largely resolved. After three months, a follow-up MRI revealed persistent findings of intracranial hypotension and his headache recurred. The decision was to repeat the epidural blood patch at the same level. However, this time $0.05 \mathrm{~mL}$ of gadolinium was added and mixed with the autologous blood to confirm the distribution of blood around the leakage site. A total of $12 \mathrm{ml}$ of blood was injected. A MRI performed immediately after the epidural blood patch showed a satisfactory spread of blood to the site of leakage (Figure 3A,B).

A follow-up MRI repeated four months later showed the disappearance of abnormal radiological features associated with intracranial hypotension. The patient remained asymptomatic one year following the second epidural blood patch.

\section{DiscuSSION}

This case report demonstrates the use of intrathecal gadolinium for the localization of high cervical CSF leakage for $\mathrm{SIH}$, the successful non-surgical treatment of this condition with a fluoroscopic-guided high cervical blood patch and the potential use of gadolinium in tracking the spread of autologous blood in an epidural blood patch.

The sites of CSF leak are commonly found in thoracolumbar and cervicothoracic level. ${ }^{15,16}$ Because radionuclide cisternography has not been reliable in detecting the level of the leak, ${ }^{1,4}$ the initial approach in our institution is to perform an 

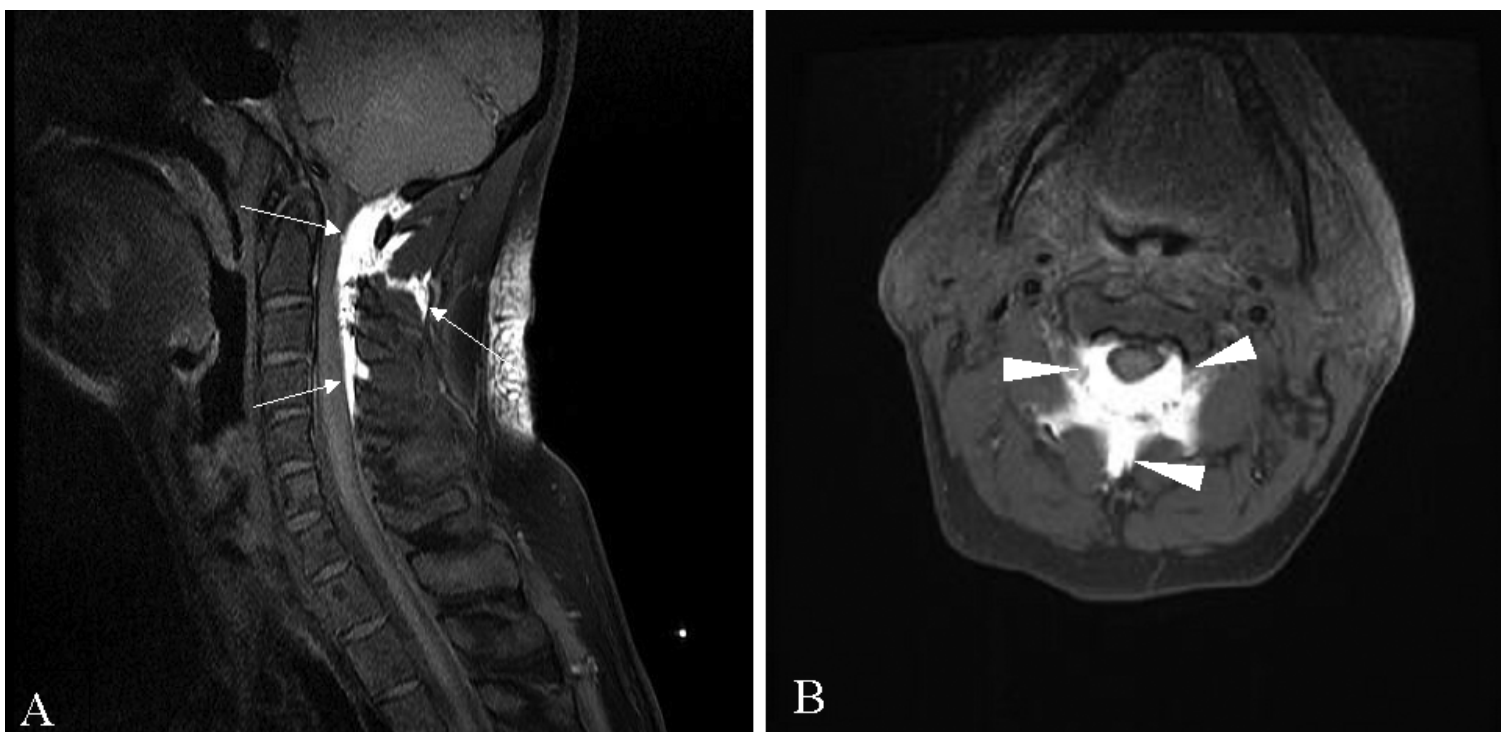

Figure 3. A and B. Sagittal and axial T1 weighted fat saturated images obtained after the second cervical epidural blood patch was performed with a small amount of gadolinium mixed in with the autologous blood. Note the hyperintense epidural blood patch extending from the opisthion to C4 and into the paraspinal tissues seen on the sagittal image (arrows in A) as well as the near circumferential epidural extent of the patch seen on the axial image (arrowheads in B).

epidural blood patch at the upper lumbar level. If the patient fails to respond, we will consider performing an epidural blood patch at the high thoracic level. In the present report there were two failed attempts to correct the leak with a 'blind' approach. Therefore, we decided to localize the site of leak with the offlabel use of gadolinium in light of our patient's history of iodinated contrast allergy. The MRI showed the presence of leakage at C1-2. Although leakage at the cervical level has been managed successfully with an epidural blood patch in literature, the levels described are at a lower cervical level. ${ }^{7,9}$ At higher levels, a surgical approach is recommended..$^{10,11}$ Concern about a high cervical epidural blood patch is related to a high rate of discontinuity of the ligamentum flavum in the cervical region. ${ }^{17}$ In this region, imaging-guided needle insertion has been suggested. ${ }^{18} \mathrm{~A}$ CT guided high cervical (C1-2) epidural blood patch has also been reported. ${ }^{12}$ However, fluoroscopy is more accessible to anesthesiologist in the operating room. In our experience, the preferred position for a high cervical epidural blood patch is the prone position. In this position, the needle entry site can be precisely kept in the midline under fluoroscopic guidance. The arm with intravenous access for sedation medication is kept beside the body and the other arm is abducted $90^{\circ}$ for venipuncture.

Presence of fluid collection in the retrospinal area does not always indicate the site of the CSF leak. In a case series of patients with spontaneous intracranial hypotension, Schievink et al reported the presence of retrospinal collection of fluid at the C1-2 level in three patients with a documented leak at lower cervical levels..$^{19}$ The retrospinal collection at C1-2 may mislead surgeons to direct surgical repair of leakage at this level.
However, in our patient, the intrathecal gadolinium could only be observed outside the spinal column at the C1-2 level. Thus, the leak was likely at the C1-2 level.

The safety of intrathecal administration of gadolinium has been well supported by the literature. In the first prospective human trial, no significant gross neurologic abnormalities, CSF changes, electroencephalographic alterations, MR morphologic evidence or MR signal changes related to the intrathecal gadolinium were seen on initial examination or on follow-up clinico-radiologic studies. ${ }^{20}$ The safety of low dose intrathecal gadolinium was further supported by a large series in humans. ${ }^{13}$ As described in our case report, the contrast agent was made further comparatively safe before subarachnoid injection by mixing a small volume of the medium with the patient's native CSF. The resultant mixture was then injected slowly to avoid hypertonic reactions of the neural tissue with the introduction of gadolinium directly into the subarachnoid space. Furthermore, the dilution of gadolinium reduces the scavenging effects of the excess chelating agent inherent in the gadolinium preparation (free dimeglumine component) for calcium ions. Thus, it reduces the possibility of acute calcium ion deficiency related neural dysfunction on the surface of the CNS.

In conclusion, intrathecal gadolinium can be a useful alternative in the localization of CSF leakage in SIH. High C1-2 CSF leakage can be successfully treatment with an epidural blood patch under fluoroscopic guidance and the addition of gadolinium to the autologous blood can be a valuable tool in examining the extent of the spread of autologous blood in the epidural space. 


\section{REFERENCE}

1. Schievink WI. Spontaneous spinal cerebrospinal fluid leaks and intracranial hypotension. JAMA. 2006; 295(19):2286-96.

2. Berroir S, Loisel B, Ducros A, Boukobza M, Tzourio C, Valade D, et al. Early epidural blood patch in spontaneous intracranial hypotension. Neurology. 2004; 63(10):1950-1.

3. Mokri B. Spontaneous cerebrospinal fluid leaks: from intracranial hypotension to cerebrospinal fluid hypovolemia-evolution of a concept. Mayo Clin Proc. 1999; 74(11):1113-23.

4. Benamor M, Tainturier C, Graveleau P, Pierot L. Radionuclide cisternography in spontaneous intracranial hypotension. Clin Nucl Med. 1998; 23(3):150-1.

5. Matsumura A, Anno I, Kimura H, Ishikawa E, Nose T. Diagnosis of spontaneous intracranial hypotension by using magnetic resonance myelography. J Neurosurg. 2000; 92(5):873-6.

6. Chiapparini L, Farina L, D'Incerti L, Erbetta A, Pareyson D, Carriero MR, et al. Spinal radiological findings in nine patients with spontaneous intracranial hypotension. Neuroradiology. 2002; 44(2):143-50.

7. Cousins MJ, Brazier D, Cook R. Intracranial hypotension caused by cervical cerebrospinal fluid leak: treatment with epidural blood patch. Anesth Analg. 2004; 98(6):1794-7.

8. Waguri N, Tomita M, Hayatsu K, Okamoto K, Shimoji K. Epidural blood patch for treatment of spontaneous intracranial hypotension. Acta Anaesthesiol Scand. 2002; 46(6):747-50.

9. Usui T, Saito S, Goto F. Spontaneous intracranial hypotension treated with a cervical epidural blood patch. Eur J Anaesthesiol. 2003;20(6): 500-2.

10. Ong B, Fong W. Spontaneous cervical spine cerebrospinal fluid leak with resultant intracranial hypotension. Clin Nucl Med. 2003; 28(11):916-9

11. Inamasu J, Nakamura Y, Orii M, Saito R, Kuroshima Y, Mayanagi $\mathrm{K}$, et al. Treatment of spontaneous intracranial hypotension secondary to C-2 meningeal cyst by surgical packing--case report. Neurol Med Chir. 2004; 44(6):326-30.
12. Rai A, Rosen C, Carpenter J, Miele V. Epidural blood patch at C2: diagnosis and treatment of spontaneous intracranial hypotension. AJNR Am J. Neuroradiol. 2006; 26(10):2663-6.

13. Tali ET, Ercan N, Krumina G, Rudwan M, Mironov A, Zeng QY, et al. Intrathecal Gadolinium (Gadopentetate Dimeglumine) enhanced magnetic resonance myelography and cisternography. Results of a multicenter study. Invest Radiol. 2002; 37(3): 152-9.

14. Jinkins JR, Rudwan M, Krumina G, Tali ET. Intrathecal Gadolinium enhanced MR Cisternography in the evaluation of clinically suspected cerebrospinal fluid rhinorrhea in humans: early experience. Radiology. 2002; 222(2):555-9.

15. Chung SJ, Kim JS, Lee MC. Syndrome of cerebral spinal fluid hypovolemia. Clinical and imaging features and outcome. Neurology. 2000; 55(9):1321-7.

16. Diaz JH. Treatment outcomes in spontaneous intracranial hypotension: do epidural blood patches stop the leaks? Pain Practice. 2004; 4(4):295-302.

17. Hogan QH. Epidural anatomy examined by cryomicrotome section. Influence of age, vertebral level, and disease. Reg Anesth. 1996; 21(5):395-406.

18. Stojanovic MP, Vu TN, Caneris O, Slezak J, Cohen SP, Sang CN. The role of fluoroscopy in cervical epidural steroid injection. An analysis of contrast dispersal patterns. Spine. 2002; 27(5):50914.

19. Schievink WI, Maya MM, Tourje J. False localizing sign of C1-2 cerebrospinal fluid leak in spontaneous intracranial hypotension. J Neurosurg. 2004; 100(6):639-44.

20. Zeng Q, Xiong L, Jinkins JR, Fan Z, Liu Z. Intrathecal gadolinium (gadopentetate dimeglumine)-enhanced MR myelography: a pilot study in human patients. Am J Radiol. 1999; 173(4): 1109-15. 